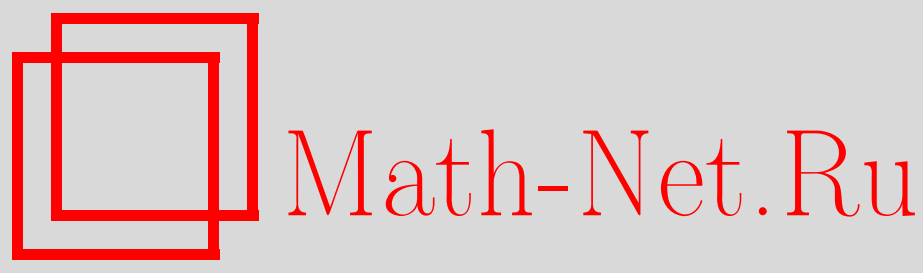

Л.Н.Липатов, Эффективное действие для реджевских процессов в квантовой хромодинамике и гравитации, ТМФ, 2011, том 169, номер 1, 9-19

DOI: https://doi.org/10.4213/tmf6704

Использование Общероссийского математического портала Math-Net.Ru подразумевает, что вы прочитали и согласны с пользовательским соглашением http://www . mathnet.ru/rus/agreement

Параметры загрузки:

IP : 54.224 .135 .184

26 апреля 2023 г., 14:08:15

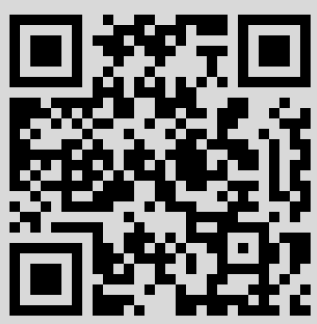




\title{
(C) 2011 г. \\ ЭФФЕКТИВНОЕ ДЕЙСТВИЕ ДЛЯ РЕДЖЕВСКИХ ПРОЦЕССОВ В КВАНТОВОЙ ХРОМОДИНАМИКЕ И ГРАВИТАЦИИ
}

\begin{abstract}
Рассматривается грибовский подход к высокоэнергетическому адрон-адронному рассеянию. Он базируется на эффективной теории поля, описывающей взаимодействия померонов. Глюоны и гравитоны реджезуются в квантовой хромодинамике и в гравитации, в связи с чем представляется естественным переформулировать эти теории при высоких энергиях в терминах реджеонов. Изложены основные идеи подхода Балицкого, Фадина, Кураева и Липатова в квантовой хромодинамике и в суперсимметричных моделях; этот подход представлен в виде калибровочно-инвариантной эффективной теории для реджезованных глюонных взаимодействий. Аналогичное общековариантное действие для реджезованных гравитонов формулируется в терминах эффективных токов, удовлетворяющих уравнению Гамильтона-Якоби.
\end{abstract}

Ключевые слова: амплитуды рассеяния, асимптотики Редже, уравнение Балицкого-Фадина-Кураева-Липатова, эффективное действие, квантовая гравитация.

\section{1. ВВЕДЕНИЕ}

Высокоэнергетическое рассеяние элементарных частиц играет важную роль, обеспечивая возможность исследовать экспериментально их внутреннюю структуру при больших передаваемых импульсах $Q$ (в глубоконеупругих лептон-адронных процессах) и структуру обмениваемых частиц (в реджевских процессах). В обоих случаях составная структура адронов наблюдалась еще до открытия квантовой хромодинамики (КХД). С помощью механизма асимптотической свободы можно построить $Q^{2}$-эволюцию партонных распределений в рамках уравнений Докшицера-Грибова-Липатова-Альтарелли-Паризи [1]. Другой подход к описанию глубоконеупругого рассеяния основан на ренормализационной группе и разложении операторных произведений, в которых структурные функции строятся в виде суммы матричных элементов операторов твиста 2, выраженных в терминах их аномальных размерностей [1]. Наиболее общий поход к реджевским процессам в КХД формулируется в терминах реджезованных глюонов и их взаимодействий. Он связан с тем обстоятельством, что амплитуды рассеяния с цветовым октетом в $t$-канале имеют реджевский вид. Процессы с бесцветными квантовыми числами возникают в результате

* Петербургский институт ядерной физики им. Б. П. Константинова РАН, Гатчина, Ленинградская обл., Россия. E-mail: lipatov@mail.desy.de 
мультиреджеонного обмена в $t$-канале [1]. Гравитон в квантовой гравитации также реджезуется. В некоторых суперсимметричных моделях реджезованный гравитон проявляет свойства померона Балицкого-Фадина-Кураева-Липатова (БФКЛ), описывающего высокоэнергетические адрон-адронные взаимодействия в КХД [1], и поэтому представляется интересным исследовать высокоэнергетические процессы рассеяния в гравитации. Ниже будут построены эффективные теории поля, описывающие взаимодействия реджезованных глюонов и гравитонов с обычными кварками, глюонами и гравитонами.

\section{2. ПОЛЮСЫ РЕДЖЕ И ГРИБОВСКИЙ ПОДХОД}

В кинематике Редже,

$$
s=4 E^{2} \gg-t=\vec{q}^{2},
$$

парциальное волновое разложение в $t$-канале для амплитуд упругого рассеяния с определенной сигнатурой $p= \pm 1$ имеет вид

$$
A^{p}(s, t)=s \int_{a-i \infty}^{a+i \infty} \frac{d \omega}{2 \pi i}\left((-s)^{\omega}-p s^{\omega}\right) f_{\omega}^{p}(t) .
$$

В рамках гипотезы реджевских полюсов для парциальной волны в $t$-канале справедливо представление

$$
f_{\omega}^{p}(t)=\frac{\gamma^{2}(t)}{\omega-\omega_{p}(t)}
$$

где $\omega_{p}-$ реджевская траектория, которая, как предполагается, линейна по $t$ :

$$
\omega_{p}(t)=\Delta-\alpha^{\prime} \vec{q}^{2}
$$

В такой модели амплитуда рассеяния имеет реджевский вид [2]:

$$
A_{\text {Regge }}^{p}(s, t)=\xi_{p}(t) s^{1+\omega_{p}(t)} \gamma^{2}(t), \quad \xi_{p}(t)=e^{-i \pi \omega_{p}(t)}-p .
$$

В работе [3] показано, что полюсы Редже порождают сингулярности типа разрезов в $\omega$-плоскости. Например, в случае двухреджеонного обмена получим следующее выражение для мандельштамовского вклада:

$$
A_{\mathrm{Mand}}^{p}(s, t)=\xi_{p} s \int \frac{d^{2} k}{(2 \pi)^{2}} \Phi^{2}(k, q-k) s^{\omega_{p_{1}}\left(-k^{2}\right)} s^{\omega_{p_{2}}\left(-(q-k)^{2}\right)} .
$$

В соответствии с грибовским законом сохранения сигнатуры получим

$$
p=p_{1} p_{2}
$$

В работе [4] доказано, что мандельштамовские разрезы возникают в результате аналитического продолжения многочастичного соотношения унитарности в $t$-канале:

$$
\operatorname{Im}_{t} f_{\omega}(t) \sim \sum_{n} \int d \Omega_{n}\left|f_{\omega}^{(n)}\right|^{2}
$$


Чтобы описать примерно постоянное поведение полного сечения рассеяния $\sigma$, связанного с амплитудами упругого рассеяния с помощью оптической теоремы, вводится специальный реджеон - померон с вакуумными квантовыми числами:

$$
\sigma=\frac{1}{s} \operatorname{Im} A(s, 0), \quad A(s, t) \approx i s s^{\Delta-\alpha_{P}^{\prime} q^{2}},
$$

где пересечение траектории (интерсепт) $\Delta$ мало.

Рожденные и виртуальные частицы при высоких энергиях объединяются в кластеры, которые сильно отличаются друг от друга значениями их быстрот

$$
0<y_{1}<y_{2}<\cdots<y_{k}<\ln s, \quad y_{k}-y_{k-1} \gg 1, \quad y_{k}=\frac{1}{2} \ln \frac{E_{k}+\left|p_{k}\right|}{E_{k}-\left|p_{k}\right|} .
$$

Кластер частиц в грибовской картине взаимодействует с другими кластерами посредством обмена померонами. Функция Грина померона в линейном приближении для его реджевской траектории имеет нерелятивистский вид:

$$
G_{0}=\frac{1}{E+\Delta-q^{2} /(2 m)}, \quad E=-\omega, \quad \alpha^{\prime}=\frac{1}{2 m} .
$$

Отсюда получается эффективная реджеонная теория поля в размерности $2+1$, задаваемая грибовским действием [5]

$$
S=\int d y d^{2} \rho\left(\phi^{*}\left(\partial_{y}-\Delta\right) \phi+\frac{1}{2 m}\left|\partial_{\mu} \phi\right|^{2}+i \lambda\left(\phi^{*} \phi^{2}+\phi \phi^{* 2}\right)+\cdots\right) .
$$

\section{3. РЕДЖЕЗАЦИЯ ГЛЮОНОВ И МУЛЬТИРЕДЖЕОННЫЕ ПРОЦЕССЫ}

Борновская амплитуда упругого рассеяния в реджевском пределе для КХД имеет вид

$$
\left.M_{A B}^{A^{\prime} B^{\prime}}(s, t)\right|_{\text {Born }}=g T_{A^{\prime} A}^{c} \delta_{\lambda_{A^{\prime}} \lambda_{A}} \frac{2 s}{t} g T_{B^{\prime} B}^{c} \delta_{\lambda_{B^{\prime}} \lambda_{B}},
$$

отвечающий закону сохранения спиральностей $\lambda$ сталкивающихся частиц $A$ и $B$. В ведущем логарифмическом приближении (ВЛП), в котором

$$
\alpha_{s} \ln s \sim 1, \quad \alpha_{s}=\frac{g^{2}}{4 \pi} \ll 1,
$$

амплитуда содержит множитель Редже:

$$
M_{A B}^{A^{\prime} B^{\prime}}(s, t)=\left.M_{A B}^{A^{\prime} B^{\prime}}(s, t)\right|_{\text {Born }} s^{\omega(t)},
$$

где реджевская траектория глюона в ВЛП имеет вид

$$
\omega\left(-|q|^{2}\right)=-\frac{\alpha_{s} N_{c}}{4 \pi^{2}} \int d^{2} k \frac{|q|^{2}}{|k|^{2}|q-k|^{2}} \approx-\frac{\alpha_{s} N_{c}}{2 \pi} \ln \frac{\left|q^{2}\right|}{\lambda^{2}} .
$$

В мультиреджевской кинематике, в которой энергии пар $\sqrt{s_{k}}$ рождаемых глюонов велики по сравнению с передаваемыми импульсами $q_{i}$ и с поперечными компонентами $k_{r \perp}$ импульсов рождаемых частиц, амплитуда рассеяния факторизуется [6]:

$$
M_{2 \rightarrow 2+n} \sim \frac{s_{1}^{\omega_{1}}}{\left|q_{1}\right|^{2}} g T_{c_{2} c_{1}}^{d_{1}} C\left(q_{2}, q_{1}\right) \frac{s_{2}^{\omega_{2}}}{\left|q_{2}\right|^{2}} \ldots g T_{c_{n+1} c_{n}}^{d_{n}} C\left(q_{n+1}, q_{n}\right) \frac{s_{n+1}^{\omega_{n+1}}}{\left|q_{n+1}\right|^{2}},
$$


где $\omega_{i}$ - реджевские траектории, а $C\left(q_{i}, q_{i-1}\right)$ суть вершины реджеон-реджеон-глюонного взаимодействия. В ВЛП они имеют вид

$$
\omega_{r}=-\frac{\alpha_{s} N_{c}}{2 \pi}\left(\ln \frac{\left|q_{r}^{2}\right|}{\mu^{2}}-\frac{1}{\epsilon}\right), \quad C\left(q_{2}, q_{1}\right)=\frac{q_{2} q_{1}^{*}}{q_{2}^{*}-q_{1}^{*}},
$$

где предполагается, что рожденные глюоны имеют определенные спиральности. Полное сечение рассеяния можно выразить через квадраты этих амплитуд:

$$
\sigma_{t}=\sum_{n} \int d \Gamma_{n}\left|M_{2 \rightarrow 2+n}\right|^{2}
$$

Амплитуды рождения должны удовлетворять стейнмановскому требованию отсутствия одновреме́нных сингулярностей в перекрывающихся каналах:

$$
\Delta_{s_{r}} \Delta_{s_{r+1}} M_{2 \rightarrow 2+n}=0 .
$$

В соответствии с этим требованием получим следующее представление для амплитуды рождения одной частицы $M_{2 \rightarrow 3}$ в анзаце Редже:

$$
M_{2 \rightarrow 3}=c_{1}(-s)^{j\left(t_{2}\right)}\left(-s_{1}\right)^{j\left(t_{1}\right)-j\left(t_{2}\right)}+c_{2}(-s)^{j\left(t_{1}\right)}\left(-s_{2}\right)^{j\left(t_{2}\right)-j\left(t_{1}\right)} .
$$

В случае рождения двух частиц соответствующее выражение для мультиреджевской амптитуды содержит пять слагаемых:

$$
\begin{aligned}
M_{2 \rightarrow 4}= & d_{1}(-s)^{j_{3}}\left(-s_{012}\right)^{j_{2}-j_{3}}\left(-s_{1}\right)^{j_{1}-j_{2}}+d_{2}(-s)^{j_{1}}\left(-s_{123}\right)^{j_{2}-j_{1}}\left(-s_{3}\right)^{j_{3}-j_{2}}+ \\
& +d_{3}(-s)^{j_{3}}\left(-s_{012}\right)^{j_{1}-j_{3}}\left(-s_{2}\right)^{j_{2}-j_{1}}+d_{4}(-s)^{j_{1}}\left(-s_{123}\right)^{j_{3}-j_{1}}\left(-s_{2}\right)^{j_{2}-j_{3}}+ \\
& +d_{5}(-s)^{j_{2}}\left(-s_{1}\right)^{j_{1}-j_{2}}\left(-s_{3}\right)^{j_{3}-j_{2}}, \quad j_{r}=j\left(t_{r}\right) .
\end{aligned}
$$

Эти формулы можно рассматривать как дисперсионные соотношения в мультиреджевской кинематике, поскольку амплитуды рассеяния содержат вещественные и мнимые вклады. Мнимые компоненты можно построить с помощью соотношений унитарности, а вещественные компоненты получаются из приведенных выше соотношений. В ВЛП мнимые вклады в амплитуды рождения частиц $M_{m \rightarrow n}$ малы. Поэтому условия унитарности обеспечивают возможность построения замкнутых нелинейных уравнений для амплитуд, которые называются соотношениями бутстрапа. В символической форме записи они представляются в виде [6]

$$
\pi \omega\left(t_{1}\right) M_{2 \rightarrow 2+n}=\sum_{r} \operatorname{Im}_{s_{0 r}} M_{2 \rightarrow 2+n}=\sum_{t} M_{2 \rightarrow 2+t} M_{2+t \rightarrow 2+n} .
$$

Знание амплитуды $M_{2 \rightarrow 2+n}$ позволяет построить полное поперечное сечение и амплитуду рассеяния с синглетными цветовыми квантовыми числами в $t$-канале. Эту амплитуду можно представить в терминах померонной волновой функции, удовлетворяющей уравнению БФКЛ [6]:

$$
E \Psi\left(\vec{\rho}_{1}, \vec{\rho}_{2}\right)=H_{12} \Psi\left(\vec{\rho}_{1}, \vec{\rho}_{2}\right), \quad \sigma_{t} \sim s^{\Delta}, \quad \Delta=-\frac{\alpha_{s} N_{c}}{2 \pi} E_{0},
$$

где $H_{12}$ - гамильтониан БФКЛ, а $\Delta$ - параметр померонного интерсепта. В координатном представлении (представлении параметра соударения) гамильтониан записывается в виде [7]

$$
H_{12}=\frac{1}{p_{1} p_{2}^{*}}\left(\ln \left|\rho_{12}\right|^{2}\right) p_{1} p_{2}^{*}+\frac{1}{p_{1}^{*} p_{2}}\left(\ln \left|\rho_{12}\right|^{2}\right) p_{1}^{*} p_{2}+\ln \left|p_{1} p_{2}\right|^{2}-4 \psi(1),
$$


где

$$
\rho_{12}=\rho_{1}-\rho_{2}, \quad \rho_{r}=x_{r}+i y_{r}, \quad \Delta=\frac{4 \alpha N_{c} \ln 2}{\pi} .
$$

Уравнение Шредингера инвариантно относительно преобразования Мёбиуса [8]

$$
\rho_{k} \rightarrow \frac{a \rho_{k}+b}{c \rho_{k}+d},
$$

откуда получаем, что собственные значения гамильтониана $H_{12}$ нумеруются своими конформными весами

$$
m=\gamma+\frac{n}{2}, \quad \widetilde{m}=\gamma-\frac{n}{2},
$$

выраженными в терминах аномальных размерностей $\gamma=1 / 2+i \nu$ операторов твиста 2 и целочисленного конформного спина $n$. Соответствующие энергии обладают свойством голоморфной отделимости и могут быть выражены в терминах логарифмической производной $\psi(x)$ гамма-функции

$$
E=2 \chi(n, \gamma)=\psi(m)+\psi(1-m)+\psi(\widetilde{m})+\psi(1-\widetilde{m})-4 \psi(1) .
$$

Из этих свойств вытекает интегрируемость динамики БФКЛ [9], [10].

\section{4. ПОМЕРОН В $N=4$ СУПЕРСИММЕТРИЧНОЙ ТЕОРИИ ЯНГА-МИЛЛСА}

Первая поправка к ядру БФКЛ была вычислена в работе [11]. Соответствующее двухпетлевое выражение для его собственного значения имеет вид

$$
\omega=4 \hat{a} \chi(n, \gamma)+4 \hat{a}^{2} \Delta(n, \gamma), \quad \hat{a}=\frac{g^{2} N_{c}}{16 \pi^{2}},
$$

где $\chi(n, \gamma)$ - выражение Борна. Этот результат содержит неаналитические члены, пропорциональные $\delta_{n, 0}$ и $\delta_{n, 2}$. Но в случае $N=4$ расширенной суперсимметричной калибровочной теории кронекеровские символы сокращаются и двухпетлевой результат обладает свойством эрмитовой сепарабельности [12]:

$$
\Delta(n, \gamma)=\phi(M)+\phi\left(M^{*}\right)-\frac{\rho(M)+\rho\left(M^{*}\right)}{2 \hat{a} / \omega}, \quad M=\gamma+\frac{|n|}{2},
$$

где

$$
\begin{aligned}
\rho(M) & =t a^{\prime}(M)+\frac{1}{2} \zeta(2), \quad \beta^{\prime}(z)=\frac{1}{4}\left[\Psi^{\prime}\left(\frac{z+1}{2}\right)-\Psi^{\prime}\left(\frac{z}{2}\right)\right], \\
\phi(M) & =3 \zeta(3)+\Psi^{\prime \prime}(M)-2 \Phi(M)+2 \beta^{\prime}(M)(\Psi(1)-\Psi(M)), \\
\Phi(M) & =\sum_{k=0}^{\infty} \frac{(-1)^{k}}{k+M}\left(\Psi^{\prime}(k+1)-\frac{\Psi(k+1)-\Psi(1)}{k+M}\right) .
\end{aligned}
$$

Отсюда, в частности, следует, что собственное значение в $N=4$ теории обладает замечательным свойством максимальной трансцендентности [13]. При условии справедливости этого свойства удается вычислить аномальные размерности операторов твиста 2 с точностью до трех петель включительно в $N=4$ суперсимметричной теории Янга-Миллса (СЯМ) [14]. При этом важно, что $N=4$ четырехмерная конформная теория поля в соответствии с догадкой Малдасены эквивалентна суперструне, распространяющейся в 10-мерном пространстве анти-де Ситтера 
(АдС) [15]-[17]. Поэтому померон в такой модели эквивалентен реджезованному гравитону суперструнной теории.

Запишем ядро уравнения БФКЛ для $N=4$ СЯМ в приближении диффузии:

$$
j=2-\Delta-D \nu^{2}, \quad \gamma=1+\frac{j-2}{2}+i \nu
$$

где параметры $\Delta$ и $D$ зависят от константы связи. В соответствии с законом сохранения энергии-импульса имеем $D=\Delta$, а потому аномальная размерность операторов твиста 2 имеет вид

$$
\gamma=(j-2)\left(\frac{1}{2}-\frac{1 / \Delta}{1+\sqrt{1+(j-2) / \Delta}}\right) .
$$

С другой стороны, в силу соответствия АдС/КТП представление диффузии для уравнения БФКЛ отвечает линейной реджевской траектории гравитона

$$
j=2+\frac{\alpha^{\prime}}{2} t, \quad t=\frac{E^{2}}{R^{2}}, \quad \alpha^{\prime}=\frac{R^{2}}{2} \Delta .
$$

Поскольку известно поведение аномальной размерности в режиме больших констант связи, $\gamma=-\sqrt{2 \pi(j-2)} \hat{a}^{1 / 4}$, можно вычислить интерсепт померона в режиме сильной связи [14]:

$$
j=2-\Delta, \quad \Delta=\frac{1}{2 \pi} \hat{a}^{-1 / 2} .
$$

Дуальность между помероном БФКЛ и реджезованным гравитоном означает, что грибовскую померонную теорию можно описать в рамках подхода, основанного на эффективном действии для реджезованных гравитонов. Как раз по этой причине мы и рассматриваем ниже высокоэнергетические процессы рассеяния в гравитации.

\section{5. ПОДХОД ЭФФЕКТИВНОГО ДЕЙСТВИЯ В КХД}

Рассмотрим сначала эффективную теорию поля для реджезованных глюонов [18]. Соответствующее эффективное действие оказывается локальным в пространстве быстрот:

$$
y=\frac{1}{2} \ln \frac{\epsilon_{k}+|k|}{\epsilon_{k}-|k|}, \quad\left|y-y_{0}\right|<\eta, \quad \eta \ll \ln s .
$$

Можно ввести антиэрмитовы поля $v_{\mu}$, описываюшие обычные глюоны, и калибровочно-инвариантные поля $A_{ \pm}$, описывающие рождение и уничтожение реджезованных глюонов:

$$
v_{\mu}(x)=-i T^{a} v_{\mu}^{a}(x), \quad A_{ \pm}(x)=-i T^{a} A_{ \pm}^{a}(x), \quad \delta A_{ \pm}(x)=0 .
$$

Поля $A_{ \pm}$удовлетворяют уравнениям кинематических связей

$$
\partial_{-} A_{+}=\partial_{+} A_{-}=0
$$

которые выражают то обстоятельство, что параметры Судакова кластерных импульсов для частиц с примерно одинаковыми быстротами оказываются сильно упорядоченными в квазимультиреджевской кинематике. 
Эффективное действие для частиц внутри отдельного кластера имеет вид

$$
S=\int d^{4} x\left(L_{\mathrm{QCD}}+\operatorname{Tr}\left(V_{+} \partial_{\mu}^{2} A_{-}+V_{-} \partial_{\mu}^{2} A_{+}\right)\right),
$$

где $L_{\mathrm{QCD}}$ - стандартное действие КХД, а эффективные токи $V_{ \pm}$определяются следующей формулой:

$$
V_{ \pm}=-\frac{1}{g} \partial_{ \pm} P \exp \left(-\frac{g}{2} \int_{-\infty}^{x^{ \pm}} v_{ \pm}\left(x^{\prime}\right) d\left(x^{\prime}\right)^{ \pm}\right)=v_{ \pm}-g v_{ \pm} \frac{1}{\partial_{ \pm}} v_{ \pm}+\cdots
$$

Правила Фейнмана для этого действия были получены в работе [19]. Подход эффективного действия позволяет конструировать разнообразные реджеонные вершины, которые являются необходимым ингредиентом при вычислении поправок первого и второго порядков к ядру БФКЛ.

Другим приложением этой эффективной полевой теории оказывается проверка справедливости анзаца Берна-Диксона-Смирнова (БДС) [20] для амплитуд неупругого рассеяния в планарном приближении для $N=4$ СЯМ. В работах [21], [22] с применением уравнения БФКЛ для октетных цветовых состояний было показано, что амплитуду БДС $M^{2 \rightarrow 4}$ в мультиреджевской кинематике при $s, s_{2}>0, s_{1}, s_{3}<0$ необходимо умножить на фактор

$$
M^{2 \rightarrow 4}=\left(1+i \Delta_{2 \rightarrow 4}\right) M_{2 \rightarrow 4}^{\mathrm{BDS}},
$$

где в ВЛП

$$
\Delta_{2 \rightarrow 4}=\frac{a}{2} \sum_{n=-\infty}^{\infty}(-1)^{n} \int_{-\infty}^{\infty} \frac{d \nu}{\nu^{2}+n^{2} / 4}\left(V^{*}\right)^{i \nu-n / 2} V^{i \nu+n / 2}\left(s_{2}^{\delta(\nu, n)}-1\right)
$$

а $\delta(\nu, n)$ - собственное значение соответствующего гамильтониана. Величины $V$ зависят от поперечных компонент импульсов рождаемых частиц $k_{1}, k_{2}$ и от передаваемых импульсов $q_{1}, q_{2}, q_{3}$. С помощью преобразования дуальности [10] их можно представить в простом виде:

$$
V=\frac{q_{3} k_{1}}{k_{2} q_{1}} \rightarrow \frac{z_{03} z_{0^{\prime} 1}}{z_{0^{\prime} 3} z_{01}}
$$

Более того, поправочный член $\Delta_{2 \rightarrow 4}$ оказывается функцией четырехмерных ангармонических отношений,

$$
i \Delta_{2 \rightarrow 4}=\frac{a^{2}}{4} \operatorname{Li}_{2}(\chi) \ln \frac{\chi t_{2} s_{13}}{s_{3} t_{1}} \ln \frac{\chi t_{2} s_{02}}{t_{3} s_{1}}+\cdots, \quad \chi=1-\frac{s s_{2}}{s_{012} s_{123}} .
$$

В двухпетлевом приближении это выражение также можно получить из свойств аналитичности и факторизации [23] или прямо из недавно полученного точного ответа для величины $M_{2 \rightarrow 4}$ [24]. С помощью эффективного действия можно построить вклады мандельштамовских разрезов, отвечающих составным состояниям с произвольным числом $n$ реджезованных глюонов в присоединенном представлении [25]. Голоморфный гамильтониан такого состояния имеет вид

$$
h=\ln \left(Z_{1}^{2} \partial_{1}\right)-2 \psi(1)+\ln \partial_{n-1}+\sum_{k=1}^{n-2} h_{k, k+1}, \quad p_{k}=Z_{k-1, k} .
$$


Гамильтониан парного взаимодействия отвечает взаимодействиям ближайших соседей в спиновой цепочке, рассмотренной в работе [9]:

$$
h_{1,2}=\ln \left(Z_{12}^{2} \partial_{1}\right)+\ln \left(Z_{12}^{2} \partial_{2}\right)-2 \ln Z_{12}-2 \psi(1) .
$$

Полный гамильтониан $h$ совпадает с гамильтонианом интегрируемой открытой спиновой цепочки [25]. Соответствующая матрица монодромии имеет вид

$$
t(u)=L_{1}(u) L_{2}(u) \cdots L_{n-1}(u)=\left(\begin{array}{ll}
A(u) & B(u) \\
C(u) & D(u)
\end{array}\right),
$$

где $L_{i}$ суть $L$-операторы, построенные из генераторов группы Мёбиуса. Матрица монодромии содержит интегралы движения и позволяет получить уравнение для функции Бакстера $Q(u)$ :

$$
[D(u), h]=0, \quad D(u) Q(u)=(u-i)^{n-1} Q(u-i) .
$$

\section{6. ЭФФЕКТИВНОЕ ДЕЙСТВИЕ ДЛЯ ГРАВИТАЦИИ}

При построении взаимодействия между реджезованными и обычными гравитонами снова можно использовать свойство локальности в пространстве быстрот (37). Различные вершины эффективного взаимодействия гравитонов и гравитонные траектории Редже в ВЛП для гравитонов были вычислены в работе [26]. В частности, было найдено, что реджевская траектория не содержит ультрафиолетовой расходимости в $N=4$ СЯМ [27]. Эффективная полевая теория для мультиреджевских процессов была построена в работе [28]. Здесь будет найдено общековариантное эффективное действие для квазимультиреджевских процессов. В общей теории относительности Эйнштейна гравитонное поле описывается метрическим тензором $g_{\mu \nu}[29]$ :

$$
d^{2} S=\sum_{\mu \nu} g_{\mu \nu} d x^{\mu} d x^{\nu}, \quad \delta g_{\mu \nu}=D_{\mu} \chi_{\nu}+D_{\mu} \chi_{\nu}
$$

где $D_{\mu}$ - ковариантные производные, а $\chi_{\nu}$ - инфинитезимальные параметры общековариантных преобразований. Реджеонные поля $A^{++}$и $A^{--}$, описывающие процессы рождения и уничтожения реджезованных гравитонов, предполагаются инвариантными относительно общековариантных преобразований с параметром $\chi_{\nu}(x) \rightarrow 0$ при $x \rightarrow \infty$. Они также удовлетворяют кинематическим условиям связей

$$
\partial_{+} A^{++}=\partial_{-} A^{--}=0 .
$$

Эффективное действие для взаимодействий этих полей, локальное относительно быстрот, имеет следующий вид:

$$
S=\frac{1}{2 \kappa} \int d^{4} x\left(-\sqrt{-g} R+j_{++} \partial_{\mu}^{2} A^{++}+j_{--} \partial_{\mu}^{2} A^{--}\right),
$$

где через $R$ обозначена риманова кривизна. Токи $j_{++}$и $j_{--}$полностью определяются условием инвариантности действия относительно общековариантных преобразований. Их можно построить с рамках теории возмущений. Например, пертурбативное разложение тока $j_{++}$имеет вид

$$
j_{++}=h_{++}-X_{\sigma+}^{2}+X_{\rho+} X_{\sigma+} h_{\rho \sigma}-X_{\sigma+} \frac{\partial_{\sigma}}{\partial_{+}} X_{\rho+}^{2}+\cdots,
$$


где

$$
g_{\mu \nu}=\eta_{\mu \nu}+h_{\mu \nu}, \quad X_{\sigma+}=h_{\sigma+}-\frac{1}{2} \frac{\partial_{\sigma}}{\partial_{+}} h_{++}
$$

и метрика Минковского $\eta_{\mu \nu}$ имеет сигнатуру $(-,+,+,+)$. Если ввести новый ток $j^{\mp}$ в соответствии с определением $j_{ \pm \pm}=\partial_{ \pm} j^{\mp}$, то он будет удовлетворять уравнению "веера"

$$
g^{\rho \mp} \partial_{\rho} j^{\mp}=-g^{\mp \mp}-\frac{1}{4} g^{\rho \sigma}\left(\partial_{\rho} j^{\mp}\right)\left(\partial_{\sigma} j^{\mp}\right) .
$$

Из вышеприведенного эффективного действия можно воспроизвести результаты работ [26], [27].

Уравнение для $j^{\mp}$ можно представить в более простом виде:

$$
g^{\rho \sigma}\left(\frac{1}{2} \partial_{\rho} j^{\mp}+g_{\rho}^{\mp}\right)\left(\frac{1}{2} \partial_{\sigma} j^{\mp}+g_{\sigma}^{\mp}\right)=0 .
$$

Его формальное решение записывается в виде

$$
j^{\mp}=-2 x^{\mp}+\omega^{ \pm}
$$

где величины $\omega^{ \pm}$удовлетворяют уравнению волнового фронта

$$
g^{\rho \sigma} \partial_{\rho} \omega^{ \pm} \partial_{\sigma} \omega^{ \pm}=0
$$

Последнее уравнение можно получить независимым образом, если искать решение общековариантно-симметричного уравнения Даламбера

$$
\nabla^{2} \phi(x)=0
$$

для волновой функции скалярной частицы, распространяющейся с большим импульсом $p^{\mp}$, в виде

$$
\phi^{ \pm}(x)=\exp \left(-i|p| x^{\mp}-i \theta^{\mp}(x)\right), \quad \theta^{\mp}(x)=\frac{|p|}{2} j^{\mp}(x) .
$$

Здесь $\theta^{\mp}(x)$ - быстро изменяющаяся фаза, а $j^{\mp}$ - эффективный ток в нашей нормировке. В самом деле, пренебрегая производными метрических тензоров по сравнению с большими величинами производных от $\phi^{ \pm}$, из уравнения Даламбера можно получить его квазиклассический предел

$$
g^{\rho \sigma}\left(\frac{1}{2} \partial_{\rho} j^{\mp}+g_{\rho}^{\mp}\right)\left(\frac{1}{2} \partial_{\sigma} j^{\mp}+g_{\sigma}^{\mp}\right)=0,
$$

который совпадает с полученным выше уравнением "веера" для $j^{\mp}$.

При фиксированном параметре соударения для частицы, рассеиваемой в гравитационном поле, в квазиклассическом приближении $S$-матрица имеет вид

$$
S=\lim _{x^{ \pm} \rightarrow \infty} \exp \left(-i \frac{|p|}{2} j^{\mp}(x)\right)
$$

в случае, если начальные условия для полей $j^{\mp}$ имеют вид

$$
\lim _{x^{ \pm} \rightarrow-\infty} j^{\mp}(x)=0 .
$$


В частности, эта $S$-матрица содержит чисто эйкональный вклад, в котором частицы промежуточных состояний лежат на массовой поверхности. Такие вклады не должны появляться в эффективном действии, хотя они и воспроизводятся итерациями эффективных взаимодействий в $s$-канале. Именно по этой причине эффективный ток $j^{\mp}$, входящий в член взаимодействия, при больших $x^{ \pm}$пропорционален логарифму $S$-матрицы:

$$
\lim _{x^{ \pm} \rightarrow \infty} j^{\mp}(x)=i \frac{2}{p^{ \pm}} \ln S .
$$

Хорошо известно [29], что решение уравнения волнового фронта

$$
g^{\rho \sigma} \partial_{\rho} \omega \partial_{\sigma} \omega=0
$$

можно выразить в терминах геодезических траекторий светоподобных частиц, удовлетворяющих уравнениям движения в гравитационном поле:

$$
\frac{d^{2} x^{\mu}}{(d \tau)^{2}}=\Gamma_{\alpha \beta}^{\mu} \frac{d x^{\alpha}}{d \tau} \frac{d x^{\beta}}{d \tau},
$$

где $\tau$ - параметр, возрастающий вдоль траектории, а $\Gamma_{\alpha \beta}^{\mu}-$ символ Кристоффеля. Уравнение геодезической можно представить в виде гамильтоновых уравнений

$$
\frac{d x^{\mu}}{d \tau}=g^{\mu \nu} \omega_{\nu}, \quad \frac{d \omega_{\alpha}}{d \tau}=-\frac{1}{2} \omega_{\mu} \omega_{\nu} \partial_{\alpha} g^{\mu \nu},
$$

где $\omega_{\alpha} \equiv \partial_{\alpha} \omega=g_{\alpha \beta} d x^{\beta} / d p$ играет роль вектора энергии-импульса.

\section{7. ЗАКЛЮЧЕНИЕ}

В настоящей работе построены эффективные действия, описывающие взаимодействие реджезованных глюонов и гравитонов с обычными частицами. Эти действия локальны относительно быстрот частиц. Из действия КХД вытекает возможность находить различные амплитуды для рождения струй в квазимультиреджевской кинематике на Большом адронном коллайдере. Также его можно использовать для вычисления поправок второго порядка к уравнению БФКЛ, описывающему высокоэнергетическое поведение полного поперечного сечения рассеяния в КХД. Эффективное действие в гравитации выражено в терминах решений уравнения волнового фронта. В рамках соответствия АдС/КТП реджезованный гравитон эквивалентен померону БФКЛ, поэтому эффективное действие для реджезованных гравитонов можно применять при проведении померонных вычислений, по крайней мере в $N=4$ СЯМ. Можно также найти поправки старших петель в гравитонную траекторию Редже и в его разнообразные члены взаимодействия. С другой стороны, с использованием реджезации гравитона в обычной теории гравитации и в расширенной теории супергравитации можно вычислять константы вычитаний в дисперсионных соотношениях для амплитуд рассеяния с помощью их аналитического продолжения из реджевской области больших $s$ и отрицательных $t$, в которой амплитуды быстро убывают, обходя таким образом проблему неперенормируемости таких моделей.

Благодарности. Автор благодарен Университету г. Гамбурга за гостеприимство и Дж. Бартелсу и А. Сабио Вере за плодотворные обсуждения. Работа частично финансировалась РФФИ (грант № 10-02-01338-а). 


\section{Список литературы}

[1] B. I. Ioffe, V.S. Fadin, L. N. Lipatov, Quantum Chromodynamics: Perturbative and Nonperturbative Aspects, Cambridge Monographs on Particle Physics, Nuclear Physics and Cosmology, 30, Cambridge Univ. Press, Cambridge, 2010.

[2] В. Н. Грибов, ЖЭТФ, 41:2 (1961), 667-669.

[3] S. Mandelstam, Nuovo Cimento, 30 (1963), 1148-1162.

[4] V. N. Gribov, I. Ya. Pomeranchuk, K. A. Ter-Martirosyan, Phys. Rev. B, 139:1B (1965), 184-202.

[5] В.Н.Грибов, ЖЭТФ, 53:2 (1967), 654-672.

[6] Л. Н. Липатов, ЯФ, 23 (1976), 642-656; V.S. Fadin, E. A. Kuraev, L. N. Lipatov, Phys. Lett. B, 60:1 (1975), 50-52; Э. А. Кураев, Л. Н. Липатов, В. С. Фадин, ЖЭТФ, 71:3 (1976), 840-855.

[7] L. N. Lipatov, Phys. Lett. B, 309:3-4 (1993), 394-396.

[8] Л.Н. Липатов, ЖЭТФ, 90:5 (1986), 1536-1552.

[9] Л.Н. Липатов, Писъма в ЖЖТФ, 59 (1994), 571-574, arXiv: hep-th/9311037.

[10] L. N. Lipatov, Nucl. Phys. B, 548:1-3 (1999), 328-362, arXiv: hep-ph/9812336.

[11] V.S. Fadin, L. N. Lipatov, Phys. Lett. B, 429:1-2 (1998), 127-134; M. Ciafaloni, G. Camici, Phys. Lett. B, 430:3-4 (1998), 349-354, arXiv: hep-ph/9803389.

[12] A. V. Kotikov, L. N. Lipatov, Nucl. Phys. B, 582:1-3 (2000), 19-43; Erratum 685:1-3 (2004), 405-407, arXiv: hep-ph/0004008.

[13] A. V. Kotikov, L. N. Lipatov, Nucl. Phys. B, 661:1-2 (2003), 19-61, arXiv: hep-ph/0208220.

[14] A. V. Kotikov, L. N. Lipatov, A. I. Onishchenko, V. N. Velizhanin, Phys. Lett. B, 595:1-4 (2004), 521-529; Erratum 632:5-6 (2006), 754-756, arXiv: hep-th/0404092.

[15] J. M. Maldacena, Adv. Theor. Math. Phys., 2 (1998), 231-252.

[16] S. S. Gubser, I. R. Klebanov, A. M. Polyakov, Phys. Lett. B, 428:1-2 (1998), 105-114, arXiv: hep-th/9802109.

[17] E. Witten, Adv. Theor. Math. Phys., 2 (1998), 253-291.

[18] L. N. Lipatov, Nucl. Phys. B, 452:1-2 (1995), 369-397, arXiv: hep-ph/9502308; Phys. Rept., 286:3 (1997), 131-198, arXiv: hep-ph/9610276.

[19] E. N. Antonov, I. O. Cherednikov, E. A. Kuraev, L. N. Lipatov, Nucl. Phys. B, 721:1-3 (2005), 111-135, arXiv: hep-ph/0411185.

[20] Z. Bern, L. J. Dixon, V. A. Smirnov, Phys. Rev. D, 72:8 (2005), 085001, 27 pp., arXiv: hep-th/0505205.

[21] J. Bartels, L. N. Lipatov, A. Sabio Vera, Phys. Rev. D, 80:4 (2009), 045002, 28 pp., arXiv: 0802.2065 .

[22] J. Bartels, L. N. Lipatov, A. Sabio Vera, Eur. Phys. J. C, 65:3-4 (2010), 587-605, arXiv: 0807.0894.

[23] L.N. Lipatov, Analytic properties of high energy production amplitudes in $\mathcal{N}=4$ SUSY, arXiv: 1008.1015.

[24] L. N. Lipatov, A. Prygarin, Phys. Rev. D, 83:12 (2011), 125001, 21 pp., arXiv: 1011.2673.

[25] L. N. Lipatov, J. Phys. A, 42:30 (2009), 304020, 25 pp.

[26] Л. Н. Липатов, 82:4 (1982), 991-1006.

[27] L. N. Lipatov, Phys. Lett. B, 116:6 (1982), 411-413.

[28] L. N. Lipatov, Nucl. Phys. B, 365:3 (1991), 614-632.

[29] V. A. Fock, The Theory of Space, Time and Gravitation, Pergamon Press, London, Aylesbury, 1969. 